\title{
AVALIAÇÃO PLASMÁTICA DE IGF-1 NO PROLACTINOMA
}

\author{
Daniela Zylberberg ${ }^{1}$, Erika C.O. Naliato 1,2, Alair Sarmet ${ }^{3}$, \\ Eduardo Sato ${ }^{4}$, Fernando S.R. Costa ${ }^{4}$, Alice H.D. Violante ${ }^{5}$
}

RESUMO - Prolactinomas são os tumores hipofisários mais comuns, podendo co-secretar GH (hormônio do crescimento). IGF-1 (fator de crescimento insulina-símile-1) é o principal responsável pelas ações do GH e parâmetro diagnóstico de acromegalia. Objetivando determinar por uma dosagem de IGF-1, na avaliação inicial de pacientes com prolactinoma, ocorrência de tumores mistos [GH e prolactina (PRL)], estudamos 7 homens e 27 mulheres, entre 19 e 72 anos, confrontando-os aos resultados de GH basal e durante teste oral de tolerância à glicose, quando $\mathrm{GH}$ basal $>0,4 \mathrm{ng} / \mathrm{mL}$ ou níveis de IGF-1 alterados. A proporção de pacientes com GH >0,4 ng/mL e IGF-1 elevada foi alta; mas, após administração de $75 \mathrm{~g}$ de glicose por via oral, nenhum paciente foi diagnosticado como acromegálico. Sugerimos, porém que a dosagem de IGF-1 seja realizada pelo risco de co-secreção de GH nos prolactinomas. Atenção especial para pacientes que apresentem significativa diminuição dos níveis de PRL, sem correspondente regressão do tamanho do adenoma.

PALAVRAS-CHAVE: fator de crescimento insulin-like l, hiperprolactinemia, prolactinoma, acromegalia.

\section{IGF-1 plasma levels evaluation in prolactinoma}

ABSTRACT - Prolactinomas are the most frequent pituitary tumors and may co-secrete GH (growth hormone). IGF-1 (insulin-like growth factor-1) is the main responsible for GH actions and a parameter for the diagnosis of acromegaly. With the objective of identifying through a IGF-1 levels analysis, in the initial evaluation of prolactinoma patients, the existence of mixed tumors [GH and prolactin (PRL)], we studied 7 men and 27 women, aged between 19 and 72 years, confronting them with the results of basal and glucose stimulated (glucose tolerance test - GTT) GH levels, indicated when GH $>0.4 \mathrm{ng} / \mathrm{mL}$ or IGF-1 levels were elevated. The prevalence of patients with $\mathrm{GH}>0.4 \mathrm{ng} / \mathrm{mL}$ and elevated IGF-1 was higher than expected; however, after GTT, no patient fulfilled the diagnostic criteria for acromegaly. However, we suggest that, they should be submitted to IGF-1 evaluation, due to the risk of GH co-secretion in prolactinomas. Special attention should be paid to those who present a significant decrease of PRL levels without concomitant tumor size reduction.

KEY WORDS: insulin-like growth factor-1, hyperprolactinemia, prolactinoma, acromegaly.

Prolactinomas, tumores secretores de prolactina (PRL), podem produzir apenas este hormônio ou adicionalmente hormônio de crescimento $(\mathrm{GH})$, hormônio adrenocorticotrófico (ACTH) ou hormônio tireotrófico (TSH), sendo a primeira, a associação mais comum. Como o uso de agonistas dopaminérgicos é o tratamento de escolha para os prolactinomas ${ }^{1,2}$, diminuindo a cada dia a indicação cirúrgica ${ }^{3}$, muitos tumores mistos podem não ser prontamente diagnosticados, por não ser feito o estudo dos mesmos por imuno-histoquímica. Realização de exames laboratoriais que possam identificar a produção conco- mitante de GH por tumores inicialmente abordados como prolactinomas seria útil para antecipar o diagnóstico de acromegalia em pacientes com tumores mistos. Fator de crescimento insulina-símile-1 (IGF-1) é o principal responsável pelas ações do $\mathrm{GH}$ e parâmetro diagnóstico de acromegalia. Produzido principalmente pelo fígado, mediante o estímulo do $\mathrm{GH}$, guarda homologia estrutural com a molécula de insulina. O IGF-1 estimula a proliferação celular e o crescimento somático ${ }^{3,4}$.

O diagnóstico bioquímico de acromegalia segue, atualmente, as diretrizes do consenso internacional

Hospital Universitário Clementino Fraga Filho, Faculdade de Medicina, Universidade Federal do Rio de Janeiro, Rio de Janeiro RJ, Brasil (FM/UFRJ): ${ }^{1}$ Mestre em Endocrinologia FM/UFRJ; ${ }^{2}$ Doutoranda do Curso de Pós-Graduação em Endocrinologia FM/UFRJ; ${ }^{3}$ Doutor em Radiologia UFRJ, Chefe do Serviço de Radiologia da Venerável Ordem $3^{\mathrm{a}}$ da Providência, Rio de Janeiro RJ, Brasil; ${ }^{4} \mathrm{Aluno}$ do Curso de Graduação da FM/UFRJ; ${ }^{5}$ Professora Adjunta Doutora e Coordenadora da Linha de Pesquisa em Hiperprolactinemia do Curso de Pós-Graduação em Endocrinologia FM/UFRJ.

Recebido 17 Março 2006, recebido na forma final 8 Junho 2006. Aceito 30 Junho 2006.

Dra. Alice H.D. Violante - Rua Domingues de Sá 289 / 601 - 24220-091 Niterói RJ - Brasil. E-mail: aliceviolante@infolink.com.br 
de 2000: se dosagens randômicas de GH e IGF-1 para idade e sexo forem normais, está excluída a doença. Caso um destes parâmetros esteja alterado, deve-se realizar a dosagem de GH basal e após 30, 60, 90 e 120 minutos da administração de 75 gramas de glicose por via oral (TOTG) 4 . O TOTG é considerado "padrão-ouro" para diagnóstico de acromegalia, que pode ser excluída quando da supressão dos níveis de $\mathrm{GH}$, a qualquer um dos tempos, para valores inferiores a $1 \mathrm{ng} / \mathrm{ml}$, mas a avaliação dos níveis de IGF-1 é hoje considerada mais sensível que GH basal para avaliação do eixo somatotrófico ${ }^{4}$.

Considerando-se a relevância crescente da dosagem de IGF-1, avaliamos a sua dosagem sérica na avaliação inicial em 34 pacientes objetivando avaliar a importância de sua dosagem rotineira na abordagem precoce do prolactinoma.

\section{MÉTODO}

Foi realizado estudo seccional com 34 pacientes consecutivos com prolactinomas que procuraram o Ambulatório de Hiperprolactinemia do Serviço de Endocrinologia do Hospital Universitário Clementino Fraga Filho - HUCFF Universidade Federal do Rio de Janeiro - UFRJ, no período de outubro de 2003 a março de 2004 . Vinte e sete mulheres $(79,5 \%)$ e sete homens $(20,5 \%)$, com idades entre 19 e 72 anos (média 36 \pm 12 ).

Nenhum paciente esteve em tratamento com agonista dopaminérgico ou qualquer outra medicação que pudesse interferir na dosagem de PRL, GH e IGF-1 por pelo menos três meses antes da realização dos exames.

Após confirmação diagnóstica de hiperprolactinemia através de duas dosagens de prolactina acima dos valores de referência propostos ("kit" Prolactin IMMULITE 2000 DPC, 2,5-17 ng/mL para homens e 2,5-20 ng/mL para muIheres) os níveis de PRL à inclusão no estudo, corresponderam ao segundo valor (confirmatório). Foram excluídas causas fisiológicas ou farmacológicas de hiperprolactinemia e realizadas as demais dosagens hormonais - hormônio folículo estimulante $(\mathrm{FSH})$, hormônio luteinizante (LH), tiroxina livre (T4L), TSH, estradiol, testosterona, GH, IGF-1 e a avaliação por imagem da região hipotálamo-hipofisária por tomografia computadorizada (TC) e/ou ressonância magnética (RM).

Os prolactinomas foram classificados em microadenomas quando o seu tamanho a avaliação neuro-radiológica (TC e/ou RM) era até $1 \mathrm{~cm}$ e macroprolactinoma quando ultrapassava este valor ${ }^{3}$.

A coleta de sangue para dosagens hormonais foi realizada no período da manhã (8 horas), após jejum de 8 a 12 horas e repouso de no mínimo 30 minutos.

Pacientes com dosagens de $\mathrm{GH}>0,4 \mathrm{ng} / \mathrm{mL}$ e/ou IGF-1 elevado para idade e sexo foram submetidos ao TOTG, com dosagem de $\mathrm{GH}$ basal e após administração de $75 \mathrm{~g}$ de glicose (Glutol $^{\circledR}$ diluído em um copo de água fria), aos 30,60 , 90 e 120 minutos. Amostras para glicemia foram coletadas nos mesmos tempos e durante todo o teste os pacientes permaneceram em repouso.

$\mathrm{Na}$ dosagem de $\mathrm{GH}$ utilizou-se um ensaio imunométrico (IMMULITE hGH ${ }^{\circledR}$ - DPC). Valores de referência de 0,06 a 5 $\mathrm{ng} / \mathrm{mL}$. Sensibilidade analítica do ensaio $0,01 \mathrm{ng} / \mathrm{mL}$, coeficiente de variação intra-ensaio, $6,5 \%$ e o coeficiente de variação interensaio, 6,2\%.

Para dosagem de IGF-1 foi usado o ensaio imunorradiométrico DSL-9400 ACTIVE ${ }^{\circledR}$ - Diagnostic Systems Laboratories, Inc, Webster, TX, USA. Sensibilidade analítica 0,03 $\mathrm{ng} / \mathrm{mL}$. Coeficiente de variação interensaio menor que $7,7 \%$ e o intra-ensaio menor que 3,3\%. Os valores de referência dependem de idade e sexo.

Como valores de cortisol basal inferiores a $10 \mu \mathrm{g} / \mathrm{dL}$ podem indicar baixa reserva hipofisária, os 11 pacientes (seis mulheres e cinco homens) com resultados abaixo deste foram submetidos ao teste de tolerância insulínica (ITT): amostras de sangue colhidas no basal e em 30, 60, 90 e 120 minutos após a administração intravenosa de 0,05 UI/Kg peso de insulina regular humana, com dosagens de glicemia, GH e cortisol. A reserva hipofisária de corticoesteróides é normal se, em presença de hipoglicemia (glicemia menor que $40 \mathrm{ng} / \mathrm{mL}$ ou $50 \%$ inferior ao valor basal), houver elevação do cortisol para níveis iguais ou superiores a 18 $\mu / d L$. Dos pacientes avaliados, apenas um apresentou resposta insuficiente do cortisol ao estímulo tendo sido iniciada reposição de corticoesteróides.

Este projeto foi aprovado pelo Comitê de Ética e Pesquisa da Faculdade de Medicina e do HUCFF/UFRJ com o número 151/04 e todos os pacientes assinaram termo de compromisso livre e esclarecido.

Análise estatística foi realizada através do programa GraphPad Prism ${ }^{\circledR}$ version 3.05 for Win 95/NT (GraphPad Software, Inc., San Diego, California - EUA). A comparação de médias foi estabelecida pelo teste t de Student. As variáveis categóricas foram comparadas pelo teste exato de Fisher e as correlações entre variáveis estudadas através da correlação linear de Pearson. Quando se fez necessário, variáveis numéricas sofreram transformação logística no intuito de permitir a utilização de testes paramétricos. Foi considerado significativo $\mathrm{p}<0,05$.

\section{RESULTADOS}

Os sintomas mais comuns entre as mulheres foram irregularidade menstrual, afetando 14 pacientes, $51,8 \%$. Sete pacientes $(25,9 \%)$ apresentavam amenorréia secundária. Onze mulheres $(40 \%)$ referiram cefaléia e dez (37\%) apresentavam galactorréia. Manifestações iniciais menos comuns eram diminuição da libido, mencionada por duas mulheres, e alteração visual, citada por apenas uma.

Nos homens, a cefaléia foi o sintoma inicial mais comum $(85,7 \%)$, seguida por redução de libido e alterações visuais $(57,1 \%)$ A disfunção erétil foi mencionada por três $(42,8 \%)$.

Dezesseis pacientes (47\%) apresentavam macro- 


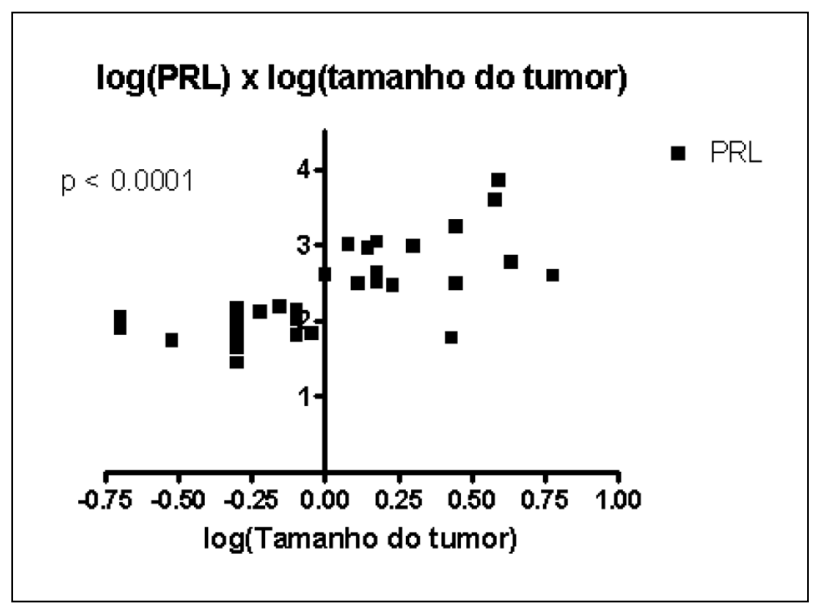

Fig 1. Correlação entre as concentrações de PRL e o tamanho dos adenomas $(p<0,0001)$.

adenomas, sendo dez mulheres e seis homens. Dezoito pacientes $(53 \%)$ tinham microadenomas.

Os valores de PRL variaram de 28 a $7000 \mathrm{ng} / \mathrm{mL}$, com média ( \pm desvio-padrão) de $632 \pm 1347,7 \mathrm{ng} / \mathrm{mL}$.

Houve correlação significativa entre as concentrações de PRL e o tamanho dos adenomas $(p<0,001)$, após transformação logarítmica de ambas as variáveis, como mostrado no Figura 1. Os homens - que em sua maioria tinham macroadenomas - apresentaram níveis séricos significativamente mais altos de PRL que as mulheres $(p=0,01)$ (Fig 2).

Nenhum paciente foi submetido à investigação de macroprolactinemia, já que em todos havia clínica de hiperprolactinemia e comprovação laboratorial e neurorradiológica de prolactinoma.

Os valores de $\mathrm{GH}$ basal variaram de 0,06 a 9,8 $\mathrm{ng} / \mathrm{mL}$, com média $0,8 \pm 1,9 \mathrm{ng} / \mathrm{mL}$. Não houve diferença estatisticamente significativa entre os sexos no que se refere aos valores de $\mathrm{GH}(\mathrm{p}=0,33)$.

Sete indivíduos apresentaram GH $>0,4 \mathrm{ng} / \mathrm{mL}$ e foram submetidos ao TOTG (Tabelas 1 e 2).

Dos 34 pacientes, apenas uma apresentava níveis de IGF-1 que pudessem ser considerados aumentados em relação à referência para sexo e idade. Não houve

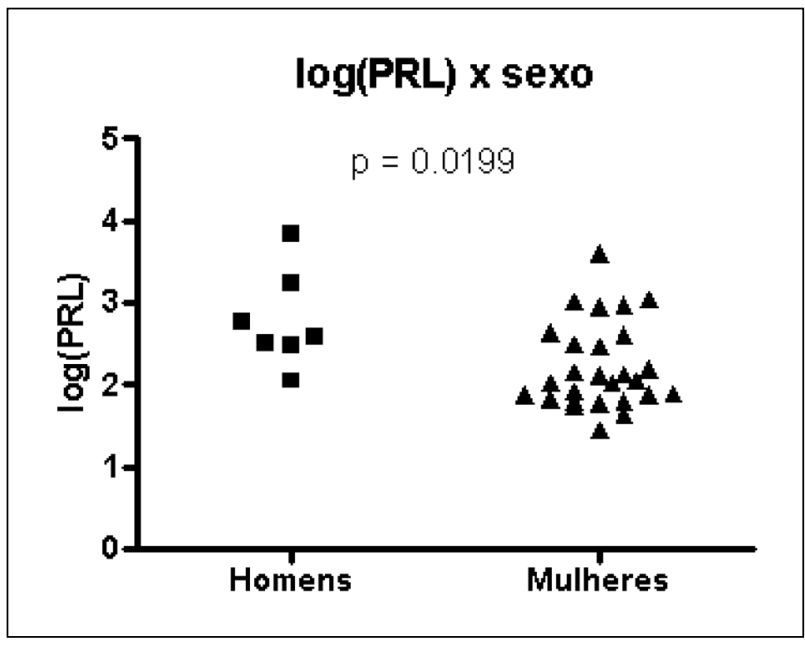

Fig 2. Correlação entre sexo e níveis de prolactina $(p=0,01)$.

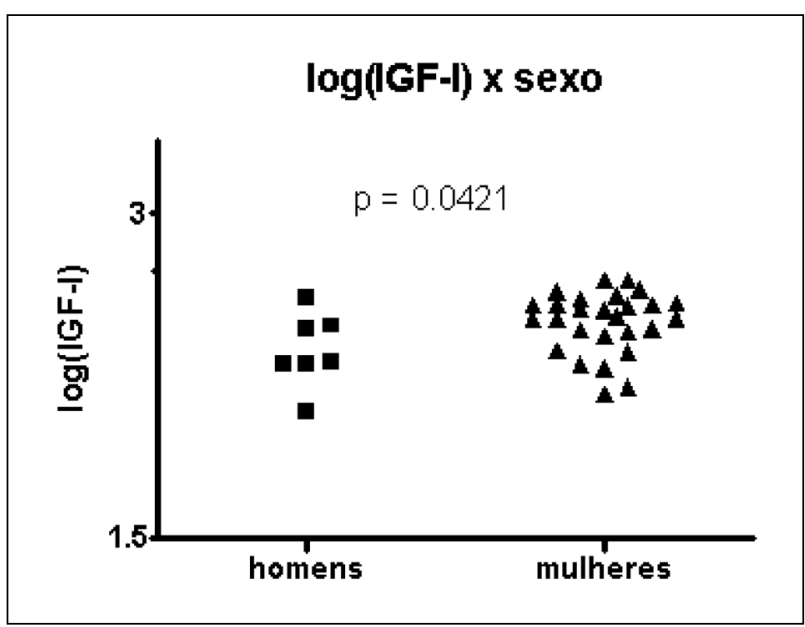

Fig 3. Correlação entre a média dos níveis de IGF-1 e sexo $(p=0,04)$.

correlação entre os níveis séricos de PRL e GH $(p=0,74)$ ou PRL e IGF-1 $(p=0,83)$. A correlação entre níveis séricos de GH e IGF-1 apenas se aproximou da significância estatística $(p=0,057)$. A média de IGF-1 das muIheres foi $2,38 \pm 0,02 \mathrm{ng} / \mathrm{mL}$ e dos homens $2,24 \pm 0,06$ $(p=0,04$, Fig 3).

Tabela 1. Níveis médios de prolactina (PRL), hormônio de crescimento (GH), fator de crescimento insulina-símile1( IGF-1) basais, teste oral de tolerância a glicose (TOTG) e tamanho do adenoma no grupo avaliado.

\begin{tabular}{lccccc}
\hline Pacientes & PRL (ng/mL) & GH & IGF-1 & $\begin{array}{c}\text { Menor medida } \\
\text { do tumor }\end{array}$ & $\begin{array}{c}\text { Maior medida } \\
\text { do tumor }\end{array}$ \\
\hline Total & $632,0 \pm 1347,7$ & $0,8 \pm 1,9$ & $241,7 \pm 82,0$ & $1,1 \pm 0,9$ & $1,5 \pm 1,4$ \\
Homens & $1497,7 \pm 2497,8$ & $0,3 \pm 0,2$ & $188,1 \pm 74,8$ & $1,8 \pm 1,3$ & $3,2 \pm 1,8$ \\
Mulheres & $407,5 \pm 789,3$ & $1,0 \pm 2,2$ & $255,5 \pm 79,2$ & $0,9 \pm 0,7$ & $1,0 \pm 0,8$ \\
\hline
\end{tabular}

Valores exibidos como média \pm desvio padrão. Valores de PRL, GH e IGF-1 expressos em ng/mL. Medidas do tumor expressas em $\mathrm{cm}$. 
Tabela 2. Níveis médios de prolactina (PRL), hormônio de crescimento (GH), fator de crescimento insulinasímile-1( IGF-1) basais, teste oral de tolerância a glicose (TOTG) e tamanho do adenoma no grupo avaliado.

\begin{tabular}{cccccccc}
\hline Paciente & Sexo & $\begin{array}{c}\text { Idade } \\
\text { (anos) }\end{array}$ & $\begin{array}{c}\text { Tamanho } \\
\text { adenoma }\end{array}$ & $\begin{array}{c}\text { PRL } \\
(1)\end{array}$ & $\begin{array}{c}\text { GH } \\
(2)\end{array}$ & $\begin{array}{c}\text { IGF-1 } \\
(3)\end{array}$ & $\begin{array}{c}\text { TOTGXGH } \\
(4)\end{array}$ \\
\hline F1 & F & 24 & micro & 138 & 37,3 & 372 & normal \\
F2 & F & 38 & micro & 120 & 1,7 & 346 & normal \\
F3 & F & 39 & macro & 255 & 9,8 & 256 & normal \\
M1 & M & 41 & macro & 7000 & 0,7 & 154 & normal \\
M2 & M & 43 & macro & 820 & 0,57 & 171 & normal \\
M3 & M & 45 & macro & 1771 & 0,5 & 230 & normal \\
F4 & F & 46 & micro & 206 & 3,14 & 218 & normal \\
F5 & F & 70 & macro & 1095 & 0,79 & 301 & normal \\
\hline
\end{tabular}

(1) Valores normais de prolactina: $2,5-17 \mathrm{ng} / \mathrm{mL}$ para homens e 2,5-20 $\mathrm{ng} / \mathrm{mL}$ para mulheres;

(2) Valores normais de $\mathrm{GH} \leq 0,4 \mathrm{ng} / \mathrm{mL}$;

(3) Valores normais de IGF-1: (Valores de referência estabelecidos por Diagnostic Systems Laboratories, Inc. Webster, Texas. Expressos em ng/mL.)

$\begin{array}{ccc}\text { Idade (anos) } & \text { Masculino } & \text { Feminino } \\ 23 \text { a } 25 & 169 \text { a } 591 & 129 \text { a } 480 \\ 31 \text { a } 40 & 100 \text { a } 494 & 130 \text { a } 354 \\ 41 \text { a } 50 & 101 \text { a } 303 & 101 \text { a } 303 \\ 50 \text { a } 70 & 78,0 \text { a } 258 & 78,0 \text { a } 258\end{array}$

(4) TOTG X GH normal: quando há supressão para níveis de GH menores que $0,4 \mathrm{ng} / \mathrm{mL}$ em pelo menos 1 momento do teste.

Todos os sete pacientes submetidos à dosagem de GH após sobrecarga de glicose apresentaram supressão para níveis menores que $1 \mathrm{ng} / \mathrm{mL}$, ou seja, não foi estabelecido diagnóstico de acromegalia em nenhum caso por meio do TOTG (Tabela 2).

\section{DISCUSSÃO}

Prolactinomas e somatotropinomas têm diferentes abordagens terapêuticas, os primeiros respondendo preferencialmente ao tratamento farmacológico com agonistas dopaminérgicos ${ }^{3,5,6}$ e os últimos tendo o tratamento cirúrgico como primeira linha ${ }^{7-10}$, a avaliação adequada do eixo somatotrófico assume grande importância no acompanhamento dos tumores inicialmente classificados como prolactinomas. Acromegalia é doença de evolução lenta e insidiosa, mas de alta morbi-mortalidade ${ }^{8,9}$. Por isso, o diagnóstico precoce da co-secreção de GH em pacientes afetados por prolactinomas deve ser sempre buscado.

A dosagem de IGF-1 é exame de fácil realização e interpretação, o que a torna uma valiosa ferramenta para o diagnóstico de acromegalia, principalmente quando aliada à dosagem de $\mathrm{GH}$, na triagem de pacientes a serem encaminhados para o TOTG com dosagem de $\mathrm{GH}^{11}$. Alguns autores consideram a dosagem de IGF-1 mais sensível que a de GH para a ava- liação do eixo somatotrófico ${ }^{3,9}$. De qualquer forma, sua utilização em populações heterogêneas mostrou que vários problemas poderiam ocorrer, quando do emprego para diagnóstico tanto de deficiência quanto de excesso de GH - duas das principais indicações para sua dosagem ${ }^{11}$.

A separação das moléculas de IGF-1 das de proteínas ligadoras de fatores de crescimento semelhante à insulina (IGFBP) e a grande variabilidade inter-e intra-ensaio constituem em importantes dificuldades analíticas na dosagem de IGF-1 ${ }^{12,13}$. A qualidade das amostras utilizadas como padrão, a coleta e armazenamento das mesmas e as diferentes afinidades individuais por diversos tipos de anticorpos conferem grande variabilidade inter-e intra-ensaio e a obtenção de amostras suficientes de material de indivíduos hígidos dentro de cada faixa etária para estabelecimento de valores de referência foi também uma importante dificuldade para os laboratórios ${ }^{14}$. $O$ ensaio mais utilizado é o imunorradiométrico (IRMA), em que podem ser procedidas técnicas com extração prévia das IGFBPs antes da leitura do material ou não ${ }^{14,15}$. Independente do método utilizado, os níveis séricos de IGF-1 podem variar com diversos fatores como idade, sexo, estado nutricional, uso de esteróides sexuais. Estudos têm avaliado variações sazonais e com etnia ${ }^{15}$. Por todos estes motivos, Giustina et al. defendem a 
interpretação dos resultados do TOTG em conjunção com os valores de IGF-1, considerando ainda que diversas condições que não a acromegalia podem ocasionar elevação de GH e que a supressão deste hormônio pode eventualmente ocorrer em acromegá$\operatorname{licos}^{4}$. Os mesmos autores acreditam que o valor de $\mathrm{GH}$ de $1 \mathrm{ng} / \mathrm{mL}$ após ingestão de glicose, atualmente utilizado para distinção entre acromegálicos e nãoacromegálicos, poderá ser redefinido no futuro, a partir da utilização de ensaios mais sensíveis 4 .

Em nosso estudo, a predominância de mulheres $(3,85: 1)$, foi menor que a relatada por outros autores, que estimam em cerca de 20:1 a proporção de prolactinomas entre os dois sexos ${ }^{16}$. Curiosamente, nossos resultados mostram também maior proporção de macroadenomas em mulheres do que a descrita para a população em geral ${ }^{16}$. Estes dois fatos podem ser justificados pelo fato do Ambulatório de Hiperprolactinemia do HUCFF / UFRJ, ser um ambulatório de referência, para onde são encaminhados os casos complexos, havendo uma pré-seleção da amostra.

Nossos dados confirmam os da literatura acerca da correlação entre tamanho do tumor e níveis de PRL e do encontro de níveis mais elevados de PRL em pacientes do sexo masculino ${ }^{16-18}$.

Clemmons et al. ${ }^{19}$, Valenta et al..$^{20} \mathrm{e}$ Yoshida et al. ${ }^{21}$ sugerem que níveis aumentados de PRL podem conduzir a valores elevados de IGF-1, independente das concentrações de GH. Entretanto, em outro estudo realizado por nosso grupo, em que incluímos somente pacientes de sexo masculino com prolactinoma cujos níveis de PRL encontravam-se muito elevados (média $\pm D P=781.2 \pm 2804.5 \mathrm{ng} / \mathrm{mL}$ ), não encontramos correlação entre níveis de PRL e IGF-122. O mesmo se deu no presente estudo. Vale a pena ressaltar que os valores de referência utilizados são estipulados para outras populações e podem não corresponder, necessariamente, à realidade da população brasileira.

O encontro de níveis discretamente mais elevados de IGF-1 em mulheres já foi mencionado na literatura, sendo explicado pela ação dos estrógenos sobre a secreção de GH, de forma direta, estimulando a liberação de hormônio hipotalâmico liberador de gonadotrofinas (GHRH), ou indireta, por inibição da liberação de somatostatina ${ }^{23}$. Parkinson et al. ${ }^{24}$, embora defendam valores de referência de acordo com idade e sexo, encontraram valores de GH basal cerca de três vezes maiores em mulheres hígidas que em homens em condições similares, mas não encontraram variação importante dos níveis de IGF-1 entre os sexos.

Como o IGF-1 tem sua síntese estimulada pelo GH, seria esperado encontrar IGF-1 aumentado nos pacientes com GH elevado e possível a ocorrência de valores baixos de $\mathrm{GH}$ em pacientes com IGF-1 muito aumentados (por "feed-back" negativo) ${ }^{24,25}$. Considerando tal hipótese, a proporção de pacientes com GH $>0,4 \mathrm{ng} / \mathrm{mL}$ e IGF-1 normal para idade e sexo foi acima da esperada (sete com $\mathrm{GH}>0,4 \mathrm{ng} / \mathrm{mL}$ e todas as IGF-1 normais).

O "screening" de hipersecreção de GH em pacientes com prolactinomas através das dosagens séricas de IGF-1 foi feito por Andersen et al. durante 5 anos, e publicado em $2003^{26}$. De 78 pacientes estudados, três com GH e IGF-1 inicialmente normais desenvolveram acromegalia clínico-laboratorial num período de 29 a 60 meses, já em uso de agonista dopaminérgico. Em apenas um houve normalização dos valores de PRL decorrente do tratamento com bromocriptina. À admissão no referido estudo, cinco outros pacientes já apresentavam IGF-1 aumentada para idade e sexo, dois dos quais não obtiveram supressão de GH ao TOTG. Nenhum deles apresentava sinais e/ou sintomas de acromegalia. Os autores supõem que mais pacientes possam ser acromegálicos e não tenham sido identificados, já que estudos anteriores sugerem que o uso de agonistas dopaminérgicos possa resultar em redução dos níveis de $\mathrm{GH}$ em pacientes com co-secreção GH-PRL ${ }^{27}$. Por este motivo, excluímos do nosso estudo pacientes que estivessem em uso de agonistas dopaminérgicos. Andersen et al. enfatizaram a importância da avaliação endócrina dos pacientes com prolactinomas, macro ou micro, sugerindo a dosagem anual de IGF-1, já que não se pode prever o intervalo necessário para o desenvolvimento de acromegalia em pacientes com prolactinomas ${ }^{26}$.

Mesmo nos casos em que já há acromegalia "estabelecida" o diagnóstico pode não ser fácil. Dimaraki et al. ${ }^{28}$ identificaram 16 acromegálicos com níveis médios de GH em 24 horas dentro dos valores propostos para "não-acromegálicos" - concentrações inferiores a $2,5 \mathrm{ng} / \mathrm{mL}$ excluiriam acromegalia, e tal dosagem só não é rotineiramente utilizada por ser pouco prática - é descrita estreita correlação com os resultados do TOTG, sendo este último de realização mais simples e, portanto, corriqueira ${ }^{4}$.

Discrepâncias entre os índices bioquímicos utilizados para diagnóstico e controle de cura na acromegalia são relatados por Abosch et al. ${ }^{29}$, e por Freda et al. ${ }^{30}$, que mostram que cerca de $13 \%$ dos pacientes mantém IGF-1 elevada após tratamento cirúrgico, a despeito de GH basal normal ou supressão ao TOTG.

Dados do presente estudo não permitem tal su- 
gestão, pois não houve confirmação de acromegalia em nenhum dos pacientes e eles não foram acompanhados prospectivamente.

Considerando os achados e comentários de Giustina et al. ${ }^{4}$, Andersen et al. ${ }^{26}$, Dimaraki et al. ${ }^{28}$, Abosch et al. ${ }^{29}$ e Freda et al. ${ }^{30}$, deverão ser submetidos a avaliações periódicas, com dosagem de IGF-1 no mínimo uma vez ao ano, aqueles pacientes que já apresentaram GH e/ou IGF-1 aumentados, em algum momento, mas também aqueles com dosagens iniciais no diagnóstico do prolactinoma dentro da normalidade, e quando há normalização ou queda significativa dos níveis de PRL sem a conseqüente diminuição no tamanho do adenoma. Recomendamos que aqueles com aumento dos níveis basais devem ser prontamente submetidos ao TOTG com dosagem de GH, visando à identificação de tumores mistos e a instituição de tratamento adequado para a acromegalia.

Concluímos que, em nosso grupo, não pudemos firmar diagnóstico de acromegalia em nenhum paciente.

Agradecimentos - Ao Laboratório Médico Sergio Franco, pela execução das dosagens de IFG-1 para este estudo.

\section{REFERÊNCIAS}

1. Biller BM. Diagnostic evaluation of hyperprolactinemia. J Reprod Med 1999;44(Suppl):S1095-S1099.

2. Molitch ME. Disorders of prolactin secretion. Endocrinol Metab Clin N Am 2001;30:585-610.

3. Aron DC, Tyrell B, Wilson CB. Pituitary tumors: current concepts in diagnosis and management. World J Med1995;162:340-352.

4. Giustina A, Barkan A, Casanueva FF, et al. Criteria for cure of acromegaly: a consensus statement. J Clin Endocrinol Metab 2000;85:526-529.

5. Foord SM, Peters JR, Dieguez C, Scalon MF, Hall R. Dopamine receptors on intact anterior pituitary cells in culture: functional association with the inhibition of prolactin and thyrotropin. Endocrinology 1983;112:567577.

6. Bronstein M. Hiperprolactinemia. In Antunes-Rodrigues,J, Moreira AC, Elias,LLK, Castro,M (eds). Neuroendocrinologia básica e aplicada. Rio de Janeiro: Guanabara-Koogan 2005:444- 455.

7. Jho, HD. Endoscopic pituitary surgery. Pituitary1999;2:139-154.

8. Turner HE. Clinical features, investigation and complications. In Handbook of acromegaly, 2003:19-28.

9. Biermasz NR, Dekker FW, Pereira AM, et al. Determinants in survival in treated acromegaly in a single center: predictive value of serial insulin-like growth factor-1 measurements. J Clin Endocrinol Metab 2004:89:2789-2796

10. Melmed S, Kleinberg D. Anterior pituitary. In Larsen PR, Kronenberg HM, Melmed S, Polanski KS (eds). Williams textbook of endocrinology, $10^{\text {th }}$ ed. Philadelphia: Saunders, 2003:177-280.
11. Clemmons DR. Commercial assays available for insulin-like growth factor- 1 and their use in diagnosis growth hormone deficiency. Horm Res 2001;55:73-79.

12. Daughday WH, Hall K, Raben MS, Salmon WD, Van der Brande ML, Van WYK JJ. Somatomedin: proposed designation for sulfation factor. Nature 1972;235:107-118

13. Thorner MO. Acromegaly and serum insulin-like growth factor-1. Horm Res 2004;62(Suppl 1):S34-S36.

14. Bang, P, Baxter RC, Blum WF, et al. Valid methods of total IGF concentrations in biological fluids: reccomendations for the 3rd International Symposium of Insulin-Like Growth Factors. J Endocrinol 1994;143: c1-c2.

15. Daughaday WH, Kapdia M, Mariz I. Serum somatomedin binding proteins: physiologic significance and interference in radiological assay. J Lab Clin Med 1987;109:355-363.

16. Mindermann T, Wilson C. Age-related and gender-related occurrence of pituitary adenomas. Clin Endocrinol 1994;41:359-364.

17. Mah PM. Hyperprolactinemia: etiology, diagnosis, and management. Semin Reprod Med 2002;20:365-374

18. Pinzone JJ, Katznelson L, Daniels DC, Pauler DK, Miller CS, Klibanski A. Primary medical therapy of micro and macroprolactinomas in men J Clin Endocrinol Metab 2000;85:3053-3057.

19. Clemmonds DR, Underwood LE, Ridgway EC, Kliman B, Van Wyk JJ Hyperprolactinemia is associated with increased immunoreactivity somatomedin-C in hypopituitarism. J Clin Endocrinol Metab 1981;52 731-735.

20. Valenta LJ, Elias AN. Clinical acromegaly with undetectable growth hormone and hyperprolactinemia. J Natl Med Ass 1987;79:555-560.

21. Yoshida T, Shimatsu A, Sakane N, Hizura N, Horikawa R, Tanaka T. Growth hormone $(\mathrm{GH})$ secretory dynamics in a case of acromegalic gigantism associated with hyperprolactinemioa: nonpulsatile secretion of GH may induce elevated insulin-like growth factor -1 (IGF-1) and IGF-binding protein-3 levels. J Clin Endocrinol Metab 1996;81:310-313.

22. Naliato ECO, Farias MLF, Braucks GR, Costa FSR, Zylberberg, D, Violante AHD. Prevalence of osteopenia in men with prolactinoma. J Endocrinol Invest 2005;28:12-17.

23. Hull KL, Harvey S. GH as a co-gonadotropin: the relevance of correlative changes in GH secretion and reproductive state. J Endocrinol 2002;172:1-19.

24. Parkinson C, Renehan AG, Ryder WDJ, Dwyert STO, Shalet SM, Trainer PJ. Gender and age influence the relationship between serum GH and IGF-1 in patients with acromegaly. Clin Endocrinol 2002;57:59-64.

25. Barkan AL, Lloyd RV, Chandler WF, et al. Treatment of acromegaly with long acting somatostatin analog SMS 201-995: shrinkage of invasive pituitary macroadenomas and improved surgical remission rate. J Clin Endocrinol Metab 1998;67:1040-1048.

26. Andersen M, Hagen C, Frystyk J, Schooder HD, Hagen K. Development of acromegaly in patients with prolactinomas. Eur J Endocrinol 2003, 149:17-22

27. Lamberts SW, Klun JG, Van Vroonhoven CC, Stefanko SZ, Liuzzi A. The role of prolactin in the inhibitory action of bromocriptine on growth hormone secretion in acromegaly. Acta Endocrinol 1983;103:446-450.

28. Dimaraki EV, Jaffe CA, De Mott-Frieberg R, Chandler WF, Barkan AL. Acromegaly with apparently normal GH secretion: implications for diagnosis and follow-up. J Clin Endocrinol Metab 2002;87:3537-3542.

29. Abosch A, Tyrell B, Lamborn K, Hannegan LT, Applebury CB, Wilson CB. Transsphenoidal microsurgery for growth hormone - secreting pituitary adenomas: initial outcome and long-term results. J Clin Endocrinol Metab 1998;83:3411-3418

30. Freda PU, Post KD, Powell JS, Wardlaw SL. Evaluation of disease status with sensitive measures of growth hormone secretion in 60 postoperative patients with acromegaly. J Clin Endocrinol Metab 1998;83:38063816. 